\title{
HÉLICES HOLÍSTICAS DE INOVAÇÃO E ECOINOVACCÃO: DRIVERS PARA O DESENVOLVIMENTO SUSTENTÁVEL
}

\author{
HOLISTIC HELIX OF INNOVATION AND ECO-INNOVATION: \\ DRIVERS FOR SUSTAINABLE DEVELOPMENT
}

\section{Eliana Andréa Severo}

Pós-Doutora em Administração pela Universidade de Caxias do Sul (Caxias do Sul/Brasil).

Professora do Mestrado Profissional em Gestão Empresarial no Centro Universitário UniFBV/Wyden (Recife/Brasil).

E-mail: elianasevero2@hotmail.com

\section{Eric Charles Henri Dorin}

Doutor em Administração de Empresas pela Universidade de Sherbrooke (Sherbrooke/Canada).

Professor do Mestrado Profissional em Gestão das Organizações Públicas na Universidade Federal

de Santa Maria (Santa Maria/Brasil).

E-mail: echdorion@gmail.com

\section{Julio Cesar Ferro de Guimarães}

Pós-Doutor em Administração pela Universidade de Caxias do Sul (Caxias do Sul/Brasil).

Professor do Departamento de Ciências Administrativas da Universidade Federal de Pernambuco (Recife/Brasil).

E-mail: juliocfguimaraes@yahoo.com.br 


\section{RESUMO}

As múltiplas hélices da inovação podem se relacionar com a ecoinovação, constituindo-se como drivers para as práticas ambientais e ações sociais que impactam as pessoas, organizações e o meio ambiente. Neste contexto, o estudo pretende avançar a compreensão sobre a relação entre as múltiplas hélices de inovação e a ecoinovação, propondo um Framework na perspectiva do desenvolvimento sustentável. A metodologia utilizada tratou-se de uma pesquisa qualitativa e exploratória, por meio de uma revisão teórica. Como resultados, destaca-se um Framework elencando-se a Proposição 1: As Hélices holísticas de inovação estão positivamente relacionadas à ecoinovação, assim como a Proposição 2: As práticas ambientais e ações sociais estão positivamente relacionadas à ecoinovação. Neste contexto, apresentamse as informações de diferentes hélices de inovação que podem ser utilizadas para fomentar uma inovação que contribua para o desenvolvimento sustentável, bem como as práticas ambientais e ações socias que visam à preservação do meio ambiente e a uma melhor qualidade de vida.

Palavras-chave: Tríplice hélice. Ecoinovação. Práticas ambientais. Responsabilidade social. Framework.

\section{ABSTRACT}

The multiple helix of innovation can relate to eco-innovation, constituting themselves as drivers for environmental practices and social actions that impact people, organizations, and the environment. In this context, the study intends to advance the understanding of the relationship between multiple innovation helix and eco-innovation, proposing a Framework for sustainable development. The methodology used was a qualitative and exploratory research, through a theoretical revision. As results, a Framework highlighting Proposition 1 stands out: The holistic helix of innovation are positively related to eco-innovation, as is Proposition 2: Environmental practices and social actions are positively related to eco-innovation. In this context, we present the information of different innovation helix that can be used to foster an innovation that contributes to sustainable development, as well as environmental practices and social actions that aim at the preservation of the environment and a better quality of life.

Keywords: Triple helix. Eco-innovation. Environmental practices. Social responsability. Framework.. 


\section{INTRODUÇÃO}

Durante as últimas décadas, as configurações das múltiplas hélices de inovação exigiram configurações para as inovações e atividades organizacionais, pois ambos os tipos de atividades empresariais desempenham um papel crucial na economia. As múltiplas hélices de inovação contribuem para a auto-organização e evolução dos sistemas de inovação, no âmbito do governo, empresas e universidades.

Neste cenário, a tríplice hélice afirma que a inovação acontece na interseção de três espaços institucionais e que há um papel importante a ser desempenhado pelas universidades. Consoante essa tríade, cada vertente relaciona-se com as outras duas, desenvolvendo uma sobreposição de comunicações, redes e organizações (DUDIN et al., 2015). Essas interações, por sua vez, são a chave para promover a inovação e o desenvolvimento econômico de países e organizações (ETZKOWITZ; LEYDESDORFF, 1995; RAZAK.; WHITE, 2015; LI et al., 2016; ETZKOWITZ; ZHOU, 2017), assim como transformam seus papéis e práticas no desenvolvimento e fortalecimento de inovação nacional e ecossistemas empreendedores (ETZKOWITZ; LEYDESDORFF, 2000). Coerentemente, o crescimento do número de empresas gera impactos positivos para o país, como aumento da competitividade e maior oferta de empregos, entretanto, requer o acompanhamento de órgãos de apoio a esses empreendimentos (NESELLO et al., 2019).

Porém, outras hélices de inovação estão emergindo no mundo contemporâneo (ETZKOWITZ; ZHOU, 2017) podendo impactar a ecoinovação com vistas ao desenvolvimento sustentável, pois os recursos naturais são finitos, bem como a superpopulação mundial e a produção industrial crescem demasiadamente.

Entretanto, problemas ambientais exigem novas soluções inovadoras (BREM; RADZIWON, 2017). Neste cenário, a literatura sobre a colaboração das múltiplas hélices relacionadas à ecoinovação ainda deixam uma lacuna de pesquisa. Guerrero e Urbano (2017) também destacam que, nas economias emergentes, os benefícios das hélices de inovação ainda são insipientes, o que requer estudos para uma melhor compreensão sobre a influência destes agentes nas inovações.

Conforme Sato (2017), esses argumentos levam a uma investigação mais aprofundada de como os casos bem-sucedidos de inovação foram alcançados somente depois que um ambiente atrativo foi criado, principalmente por meio de esforços sólidos e combinados da interação entre indústria, universidade e governo. Neste cenário, a inovação pode ser a chave, pois trata-se de um processo de aprendizagem complexo, interativo e não linear dentro de empresas e entre organizações e seu ambiente (DA SILVA et al., 2017). 
Além disso, é tópico emergente a ecoinovação junto com seus possíveis recursos na cocriação de transições sustentáveis (TRENCHER; YARIME; KHARRAZI, 2013), visto que os ecossistemas consideram principalmente grandes empresas (ROHRBECK; HÖLZLE; GEMÜNDEN, 2009; BOSCH-SIJTSEMA; BOSCH, 2015) como os representantes da indústria. Conforme Correia, Goméz e Oliveira (2018), é necessário buscar investimentos para uma mudança social, reconhecendo que os desafios futuros podem ser identificados e que existem possíveis soluções que podem ser implementadas.

Perante o exposto, a proposta da presente pesquisa é responder a seguinte questão: quais as relações entre as múltiplas hélices de inovação e a ecoinovação que visam o desenvolvimento sustentável? Coerentemente, este estudo, por meio de um ensaio teórico, tem como objetivo avançar a compreensão sobre a relação entre as múltiplas hélices de inovação e a ecoinovação, propondo um Framework na perspectiva do desenvolvimento sustentável.

\section{METODOLOGIA}

A metodologia utilizada tratou-se de uma pesquisa qualitativa e exploratória, por meio de uma revisão teórica. Conforme Flick (2009), a pesquisa qualitativa é recomendada para entender e descrever as perspectivas práticas, possibilitando experiências, interações e documentos em seu contexto natural. No que se refere ao objetivo, a pesquisa caracteriza-se como exploratória, com a finalidade de desenvolver, esclarecer e modificar conceitos e ideias, o que geralmente envolve levantamento bibliográfico e documental, proporcionando uma visão geral sobre o fato a ser estudado (GIL, 2010; CRESWELL, 2010).

Para a revisão da literatura, utilizou-se uma pesquisa sistemática na Science Direct, na qual se identificou os fatores das múltiplas hélices de inovação, bem como as práticas ambientais e ações sociais que influenciam a ecoinovação. 0 critério de escolha da Science Direct foi por conveniência, bem como é uma plataforma operada pela editora Anglo-Holandesa Elsevier, para acesso de aproximadamente 2.500 revistas científicas e cerca de 26.000 e-books.

Esta pesquisa sistemática ocorreu no dia 09 de agosto de 2018, bem como utilizou-se um filtro para verificar o número de artigos para cada palavra-chave: i) triple helix (tríplice hélice); ii) eco-innovation (ecoinovação); iii) triple helix and eco-innovation (tríplice hélice e ecoinovação); iv) environmental practices and eco-innovation (práticas ambientais e ecoinivação) ; e v) social responsibility and eco-innovation (responsabilidade social e ecoinovação). Neste cenário, para cada palavra-chave verificou-se os Journals com o maior número de publicações (Figura 1), além do número de artigos publicados de 2008 a 2018. Vale ressaltar que para o ano de 2018 não se tem a totalidade de artigos, visto a pesquisa ter ocorrido no mês de agosto do corrente ano. 
Para a análise e interpretação dos dados, utilizou-se a técnica de análise de conteúdo, que, segundo Bardin (2011), trata-se de um procedimento sistemático objetivando a descrição do conteúdo das mensagens, onde podem ser analisados os significados. Coerentemente, ocorreram anotações de campo e elaboração de gráficos, visando à comparação dos dados coletados, em um processo de triangulação, aumentando a validade da pesquisa (FLICK, 2009).

Na análise de conteúdo, a fim de facilitar o processo de análise e interpretação dos dados coletados, as informações foram categorizadas para trazer fidelidade na interpretação dos dados (GIBBS, 2009). As categorias foram elencadas, a priori, com base nos objetivos e palavras-chave do estudo: i) hélices de inovação; ii) ecoinovação; iii) tríplice hélice e ecoinovação; iv) práticas ambientais e ecoinovação; e v) responsabilidade social e ecoinovação.

A partir deste estudo, desenvolve-se um Framework de análise dos fatores das múltiplas hélices de inovação e da ecoinovação, que contribua para o desenvolvimento sustentável.

\section{PUBLICAÇÕES VERSUS JOURNALS}

Primeiramente, a pesquisa identificou os 10 Journals com o maior número de artigos para cada palavra-chave (Figura 1): i) tríplice hélice; ii) ecoinovação; iii) tríplice hélice e ecoinovação; iv) práticas ambientais e ecoinovação; ev) responsabilidade social e ecoinovação. Coerentemente, o destaque da tríplice hélice ocorreu no Journal of Molecular Biology, com 2.040 publicações; da tríplice hélice e ecoinovação no Journal of Cleaner Production, com 15 artigos; assim como da ecoinovação no Journal of Cleaner Production, com 1.976 publicações.

Conforme a Figura 1, ocorre destaque novamente do Journal of Cleaner Production, com o maior número de publicações, tanto para as práticas ambientais e ecoinivação, com 1.570 publicações, assim como para a responsabilidade social e ecoinovação, com 948 publicações. Consoante isso, o Journal of Cleaner Production serve como um fórum internacional interdisciplinar para o intercâmbio de informações e resultados de pesquisas sobre tecnologias, conceitos e políticas destinados a ajudar a garantir o progresso em direção a sociedades sustentáveis. Busca incentivar a inovação industrial, produtos novos e aprimorados e a implementação de novos processos e serviços mais limpos. Destina-se, também, a estimular o desenvolvimento e a implementação de políticas governamentais orientadas para a prevenção e programas educacionais. Contudo, a produção mais limpa $(P+L)$ é um conceito que vai além do simples controle da poluição.

Neste cenário, a P+L visa à otimização dos recursos utilizados no processo produtivo, diminuindo o consumo de água e energia, assim como gerando menos resíduos industriais, o que, por conseguinte, diminui os custos com o tratamento e destinação final dos resíduos, primando para a performance organizacional. 


\section{Gestãoe \\ Desenvolvimento}

Figura 1 - Journals com o maior número de artigos pulicados

\begin{tabular}{|c|c|}
\hline \multicolumn{2}{|l|}{ Tríplice hélice } \\
\hline Journal & $N^{0}$ de artigos \\
\hline Journal of Molecular Biology & 2.040 \\
\hline Biophysical Journal & 670 \\
\hline Structure & 565 \\
\hline FEBS Letters & 484 \\
\hline Cell & 355 \\
\hline International Journal of Biological Macromolecules & 353 \\
\hline Biochemical and Biophysical Research Communications & 331 \\
\hline Current Opinion in Structural Biology & 288 \\
\hline Matrix Biology & 286 \\
\hline Methods in Enzymology & 282 \\
\hline \multicolumn{2}{|c|}{ Tríplice hélice e Ecoinovação } \\
\hline Journal & $N^{\circ}$ de artigos \\
\hline Journal of Cleaner Production & 15 \\
\hline Technological Forecasting and Social Change & 12 \\
\hline Procedia - Social and Behavioral Sciences & 8 \\
\hline Technovation & 5 \\
\hline Cities & 5 \\
\hline Sustainable Cities and Society & 5 \\
\hline World Patent Information & 4 \\
\hline Fuel and Energy Abstracts & 3 \\
\hline Procedia Engineering & 3 \\
\hline City, Culture and Society & 2 \\
\hline \multicolumn{2}{|l|}{ Ecoinovação } \\
\hline Journal & $N^{\circ}$ de artigos \\
\hline Journal of Cleaner Production & 1.976 \\
\hline Energy Policy & 472 \\
\hline Energy Procedia & 422 \\
\hline Research Policy & 404 \\
\hline Renewable and Sustainable Energy Reviews & 368 \\
\hline Ecological Economics & 337 \\
\hline Futures & 297 \\
\hline
\end{tabular}




\section{Gestãoe \\ Desenvolvimento}

e-ISSN: 2446-6875

p-ISSN: 1807-5436

\begin{tabular}{|c|c|}
\hline Technological Forecasting and Social Change & 280 \\
\hline Procedia - Social and Behavioral Sciences & 280 \\
\hline Procedia CIRP & 279 \\
\hline \multicolumn{2}{|c|}{ Práticas ambientais e Ecoinovação } \\
\hline Journal & $N^{\circ}$ de artigos \\
\hline Journal of Cleaner Production & 1.570 \\
\hline Energy Policy & 263 \\
\hline Ecological Economics & 255 \\
\hline Renewable and Sustainable Energy Reviews & 202 \\
\hline Futures & 180 \\
\hline Technological Forecasting and Social Change & 174 \\
\hline Procedia CIRP & 173 \\
\hline Procedia - Social and Behavioral Sciences & 166 \\
\hline Resources, Conservation and Recycling & 150 \\
\hline Energy Procedia & 130 \\
\hline \multicolumn{2}{|c|}{ Responsabilidade social e Ecoinovação } \\
\hline Journal & $N^{\circ}$ de artigos \\
\hline Journal of Cleaner Production & 948 \\
\hline Procedia - Social and Behavioral Sciences & 272 \\
\hline Energy Procedia & 192 \\
\hline Procedia CIRP & 166 \\
\hline Energy Policy & 152 \\
\hline Futures & 133 \\
\hline Ecological Economics & 130 \\
\hline Business Horizons & 108 \\
\hline Research Policy & 102 \\
\hline Renewable and Sustainable Energy Reviews & 101 \\
\hline
\end{tabular}

Fonte: Elaborado pelos autores (2018)

\subsection{HÉLICES DE INOVAÇÃO}

A pesquisa sistemática na Science Direct para tríplice hélice encontrou 26.814 registros, isto demonstra que as hélices de inovação vêm sendo amplamente discutidas no meio científico. No que tange ao número de artigos publicados por ano (Gráfico 1), percebe-se que a partir de 2012 ocorreu um 


\section{Gestãoe \\ Desenvolvimento}

e-ISSN: 2446-6875

p-ISSN: $1807-5436$

aumento do número de artigos publicados sobre a tríplice hélice, sendo em 2017 o maior número, com 1.413 publicações científicas.

\section{Gráfico 1 - Quantidade de artigos publicados sobre tríplice hélice}

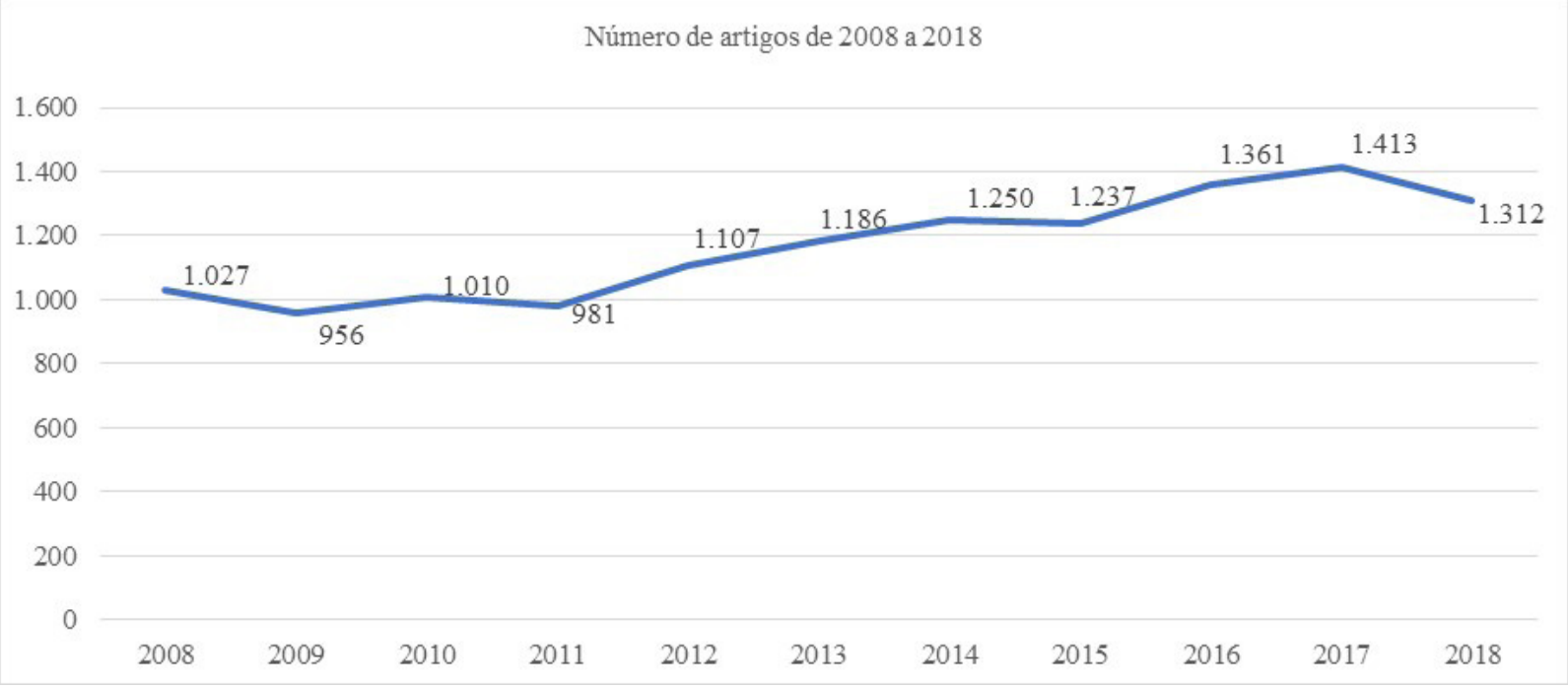

Fonte: Elaborado pelos autores (2018)

Dentre os artigos publicados, ao longo das últimas décadas, houve muita discussão sobre o papel das hélices, universidade, indústria e da colaboração governamental para o avanço da inovação e desenvolvimento econômico (ETZKOWITZ; LEYDESDORFF, 1999; ETZKOWITZ, 2003a, 2003b; LI et al., 2016). Lee e Kim (2016) ressaltam que as interações ativas nas redes de pesquisa e desenvolvimento (P\&D) dos atores institucionais da tríplice hélice, como universidade-indústria-governo, podem melhorar as capacidades inovadoras dos países.

Neste contexto, as universidades têm um papel fundamental para as relações das hélices da inovação, pois formam cidadãos que irão gerenciar as organizações (LIU; HUANG, 2018). Para Brem e Radziwon (2017), a colaboração entre as universidades e as partes interessadas locais está se tornando um fator chave de sucesso para o crescimento de ecossistemas empresariais regionais, contribuindo para a inovação.

De acordo com Liu e Huang (2018), os governos devem reconhecer e prestar muita atenção à universidade, bem como na sua capacidade e papel na inovação para o desenvolvimento regional. Neste cenário, o governo tem uma ação significativa ao criar e manter a estrutura, na qual as empresas possam inovar, pois define e regula o processo de inovação das empresas (VAIVODE, 2015). 


\section{Gestãoe \\ Desenvolvimento}

e-ISSN: 2446-6875

p-ISSN: $1807-5436$

Conforme Ryan, Geoghegan e Hilliard (2018), o governo compromete recursos significativos, apoio e direção para a colaboração universidade-indústria para facilitar o sistema de produção de conhecimento para as empresas, objetivando aumentar o crescimento econômico e o valor social.

Para Sato (2017), no centro do debate sobre a importância governamental está o Japão, com suas políticas holísticas inovadoras, bem como os novos participantes que já estão impulsionando significativos impactos da tríplice hélice, como representantes do Oriente Médio, uma região onde a sustentabilidade e a inovação têm componentes-chave dos esforços em curso para transformar as áreas desérticas, outrora pouco povoadas, em cidades globais e smart cities. Conforme Nilssen (2018), o conceito de smart cities elenca as habilidades de cidades a serem inovadoras, melhorando a qualidade de vida urbana.

\subsection{ECOINOVAÇÃO}

A pesquisa sistemática para ecoinovação encontrou 21.392 registros, isto demonstra que a ecoinovação está em pauta no meio científico. Para o número de artigos publicados por ano, o Gráfico 2 ressalta que em 2017 ocorreu o maior número, com 2.554 publicações científicas.

A ecoinovação vem sendo amplamente discutida na literatura, assim como apresenta diferentes expressões relacionadas a mesma abordagem ou assunto, por exemplo, inovações sustentáveis, inovações ecológicas, inovações verdes, inovações ambientais (HALILA; RUNDQUIST, 2011; DÍAZ-GARCÍA; GONZÁLEZ-MORENO; SÁEZ-MARTÍNEZ, 2015; LI et al., 2018; CHEN et al., 2018) e ecoinovações, sendo este último o termo principal usado neste artigo. A ecoinovação é transdisciplinar (SANTOLARIA et al., 2011; BOONS; LÜDEKE-FREUND, 2013; BOSSLE et al., 2016).

\section{Gráfico 2 - Quantidade de artigos publicados sobre ecoinovação}

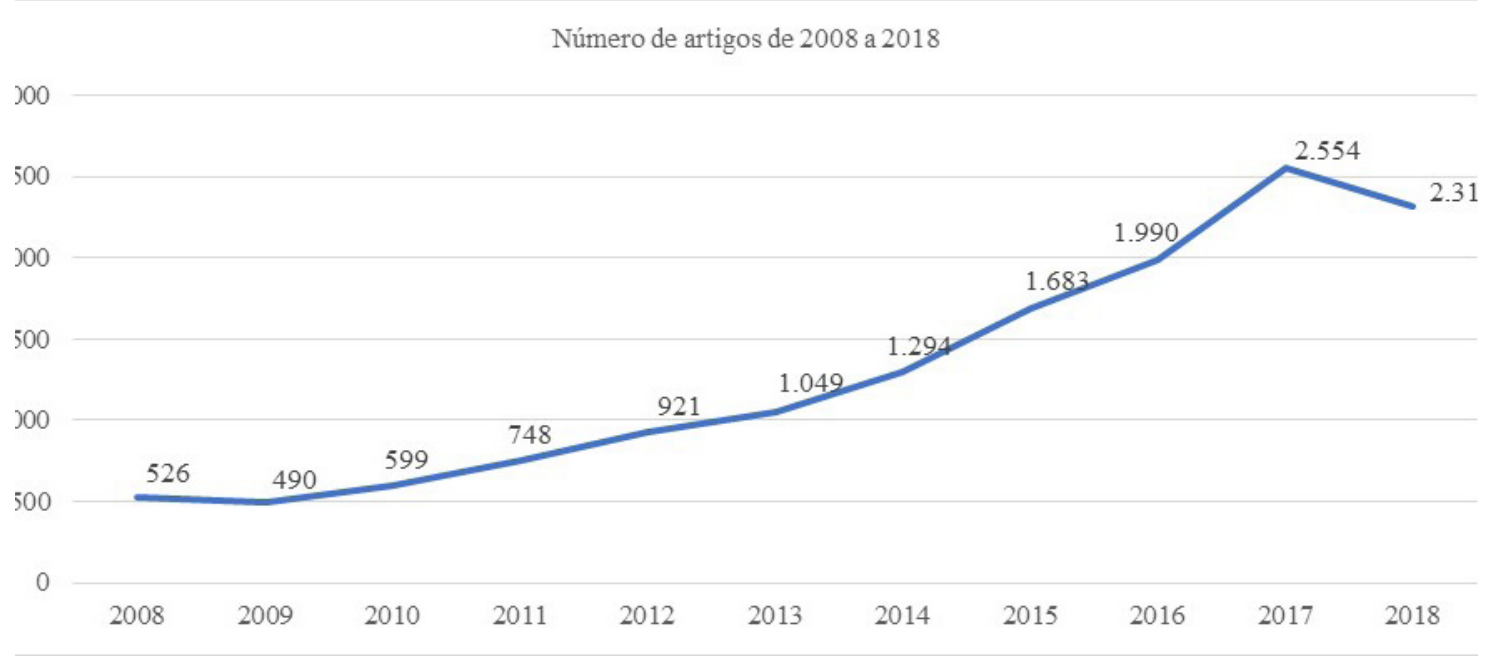

Fonte: Elaborado pelos autores (2018) 


\section{Gestãoe \\ Desenvolvimento}

e-ISSN: 2446-6875

p-ISSN: $1807-5436$

Neste contexto, a literatura sobre ecoinovação fornece contribuições extensivas para a obtenção de resultados da sustentabilidade a longo, o que implica uma necessidade de mudanças holísticas em torno dos processos de negócios. Neste sentido, vários modelos têm sido propostos para ajudar as empresas a alcançar uma maior compreensão da dinâmica da ecoinovação ou mesmo estruturar e facilitar a integração de processos sustentáveis entre eles (XAVIER et al., 2017). Ainda conforme os autores, poucos são os modelos com nível detalhado, bem como ocorre a falta de modelos relacionados a fatores estruturais da empresa (habilidades específicas, capacidade ambiental, cultura, liderança), de modelos relacionados aos aspectos sociais da sustentabilidade, de modelos com foco no serviço, e também de modelos que refletem o alto potencial de inovação sustentável da organização.

Segundo Pialot e Millet (2018), as consequências ambientais do consumo em massa exigem a ecoinovação, o que significa repensar completamente a nossa maneira de projetar, fabricar e consumir, propondo no mercado produtos e serviços com alta ambição ambiental.

Portanto, a importância de incluir todos os interessados na transição para uma economia que integra conceitos ecológicos em estratégias de inovação e competitividade é destacada na adopção de uma estratégia de ecoinovação (BOSSLE et al., 2016).

\subsection{TRÍPLICE HÉLICE E ECOINOVAÇÃO}

No que se refere à tríplice hélice e ecoinovação, a pesquisa encontrou um total de 137 artigos publicados. Neste contexto, percebe-se que a tríplice hélice associada à ecoinovação carece de pesquisas conjuntas, pois são usualmente publicadas separadamente.

O Gráfico 3 destaca o número de artigos publicados por ano. Contudo, o ano de 2018 é o de maior publicação, com 32 artigos científicos (mesmo com a pesquisa sendo realizada em agosto, ou seja, a não totalidade do ano). Estes resultados indicam que a partir de 2017 evoluíram as publicações com a associação dos dois temas. 


\section{Gráfico 3 - Quantidade de artigos publicados sobre tríplice hélice e ecoinovação}

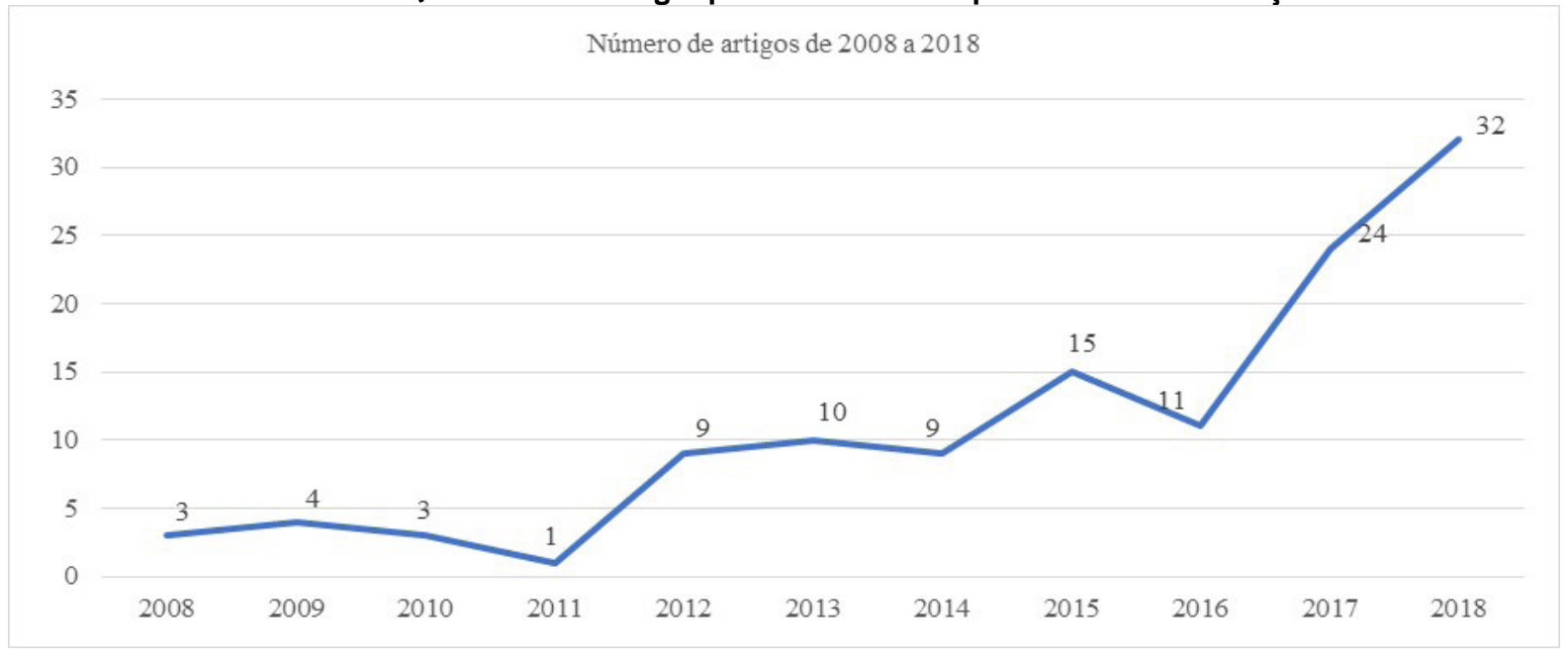

Fonte: Elaborado pelos autores (2018)

De acordo com Cancino et al. (2018), ocorre uma necessidade de gerenciar inovações tecnológicas para o crescimento sustentável a partir de uma perspectiva sistemática. Tamayo-Orbegozo, VicenteMolina e Villarreal-Larrinaga (2017) ressaltam que a ecoinovação está atraindo crescente interesse entre as empresas, governos e pesquisadores como um meio de alcançar um grau mais elevado de desenvolvimento sustentável.

Neste cenário, a tríplice hélice pode fomentar a ecoinovação (CARAYANNIS; CAMPBELL, 2010; GOUVEA; KASSICIEH; MONTOYA, 2013), ou seja, uma inovação que contribua para o desenvolvimento sustentável, a fim de preservar os recursos naturais para as futuras gerações (SEVERO; GUIMARÃES, DORION, 2018).

A tríplice hélice e a ecoinovação são frequentemente estruturas aplicadas para analisar como as preocupações ambientais são integradas nos processos de inovação e como os diferentes grupos de partes interessadas estão interagindo nessa conexão (YANG; HOLGAARD; REMMEN, 2012).

Para Carayannis e Campbell (2009), existe uma quarta hélice, a qual combina na perspectiva de um público baseado na mídia e na cultura, resultando em um ecossistema de conhecimento e inovação emergente, bem configurado para a economia e sociedade do conhecimento. Conforme Carayannis, Cherepovitsyn e llinova (2017), a quinta hélice apoia a formação de uma situação ganha-ganha entre ecologia, conhecimento e inovação, criando sinergias entre economia, sociedade e democracia, que é a boa base para o desenvolvimento sustentável dos territórios.

As políticas inovadoras, por mais revolucionárias que sejam, não serão bem-sucedidas, a menos que o mercado e seus consumidores estejam prontos para desempenhar um papel significativo como 


\section{Gestãoe \\ Desenvolvimento}

e-ISSN: 2446-6875

p-ISSN: $1807-5436$

adotantes. Neste sentido, os líderes do governo podem se tornar, primeiramente, catalisadores-chave para a inovação através da implementação de incentivos públicos que apoiem novos conceitos e ideias (SATO, 2017). Por conseguinte, isso pode resultar em uma nova geração de produtos, serviços e tecnologias verdes capazes de estimular a economia mundial (GOUVEA; KASSICIEH; MONTOYA, 2013).

\subsection{PRÁTICAS AMBIENTAIS E ECOINOVAÇÃO}

Para as práticas ambientais e ecoinovação, a pesquisa encontrou um total de 10.350 artigos publicados. Quanto ao número de artigos publicados por ano, o Gráfico 4 apresenta que o ano de 2017 foi quando ocorreu o maior número, com 1.301 publicações científicas. Neste cenário, identifica-se que o número de publicações foi aumentando gradativamente com o passar dos anos.

\section{Gráfico 4 - Quantidade de artigos publicados sobre práticas ambientais e ecoinovação}

Número de artigos de 2008 a 2018

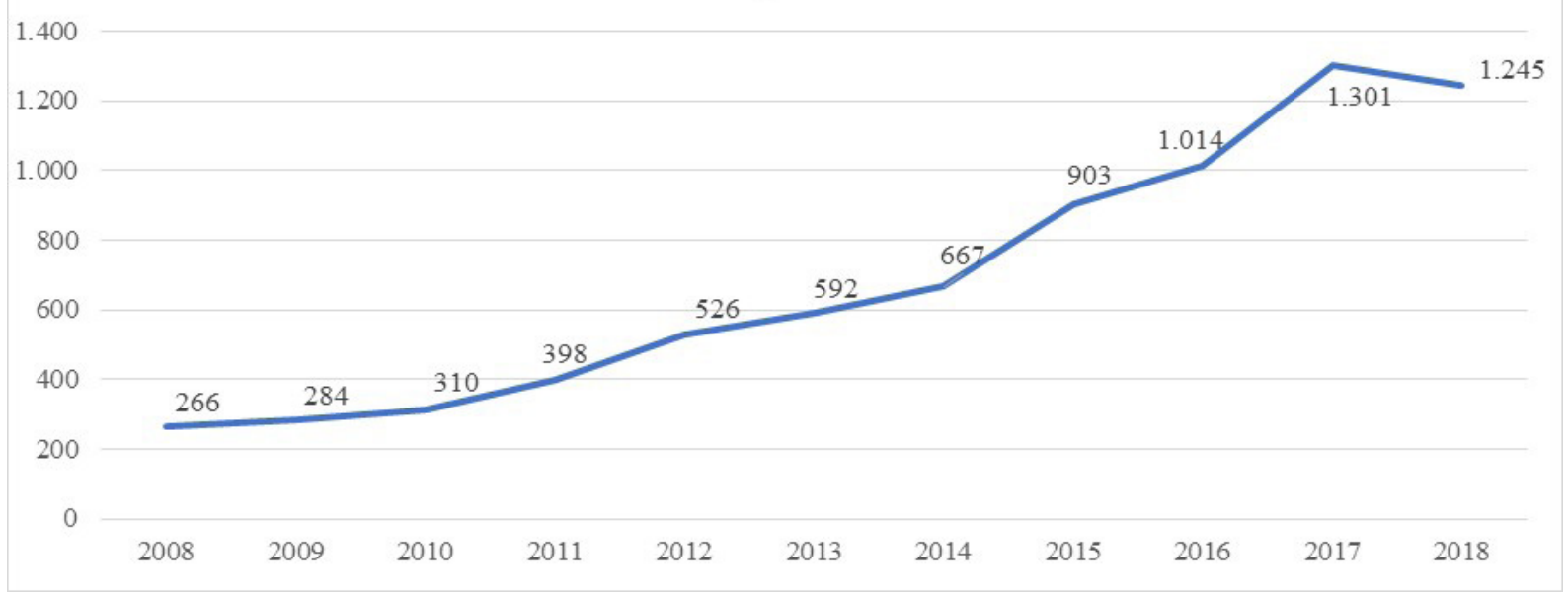

Fonte: Elaborado pelos autores (2018)

Nos últimos anos, o tema de práticas ambientais, por meio da ecoinovação, tem recebido crescente atenção na pesquisa acadêmica (CHEN; CHENG, DAI, 2017; CAI; LI, 2018; HOJNIK; RUZZIER; MANOLOVA, 2018) e nos círculos políticos (HOJNIK; RUZZIER, 2016). Conforme Chen, Cheng e Dai (2017), nesta nova era da civilização ecológica, a ecoinovação tem um alto e distintivo valor para as organizações contemporâneas. Hojnik, Ruzzier e Manolova (2018) ressaltam que está em ascensão a adoção de ecoinovação, tanto pelas empresas, quanto pelos consumidores. A nível industrial, o desenvolvimento de ecoinovação constitui um mecanismo para alcançar a sustentabilidade (LOPEZ; MONTALVO, 2015).

Neste contexto, a implementação das práticas ambientais associadas à ecoinovação está posicionada como um alvo para que as organizações sejam mais sustentáveis, a fim de reduzir as externalidades 


\section{Gestãoe \\ Desenvolvimento}

e-ISSN: 2446-6875

p-ISSN: $1807-5436$

negativas e exigências ecológicas dos governos e as exigências dos consumidores (GARCÍA-GRANERO; PIEDRA-MUÑOZ; GALDEANO-GÓMEZ, 2018).

Segundo Cai e Li (2018), a pressão competitiva fornece às empresas um maior incentivo para implementar a ecoinovação. Conforme Hojnik e Ruzzier (2016), os regulamentos e os fatores de atração do mercado são os impulsionadores mais críticos da ecoinovação nas empresas.

As práticas ambientais adjuntas à ecoinovação projetam um novo sistema integrando as dimensões do desenvolvimento sustentável, elencando o meio ambiente, as questões sociais, a tecnologia e os stakeholders (PIALOT; MILLET, 2018). Coerentemente, as práticas ambientais por meio da ecoinovação visam à redução do uso de recursos naturais, tais como materiais, energia, água e terra, bem como a diminuição de liberação de substâncias nocivas através da introdução de um novo ou melhorado produto (bem ou serviço), processo, mudança organizacional (CHENG; SHIU, 2012; CHENG; YANG; SHEU, 2013), ou programas de mercado (CHEN; CHENG; DAl, 2017).

\subsection{RESPONSABILIDADE SOCIAL E ECOINOVAÇÃO}

A pesquisa para responsabilidade social e ecoinovação encontrou 6.823 registros, isto demonstra que as ações sociais associadas à ecoinovação apresentam menor número de artigos, quando comparadas com as práticas ambientais e ecoinovação. No âmbito do número de artigos publicados por ano (Gráfico 5), identifica-se que a partir de 2014 ocorreu um aumento do número de artigos publicados sobre as temáticas, sendo em 2017 o maior número, com 811 publicações científicas.

\section{Gráfico 5 - Quantidade de artigos publicados sobre responsabilidade social e ecoinovação}

Número de artigos de 2008 a 2018

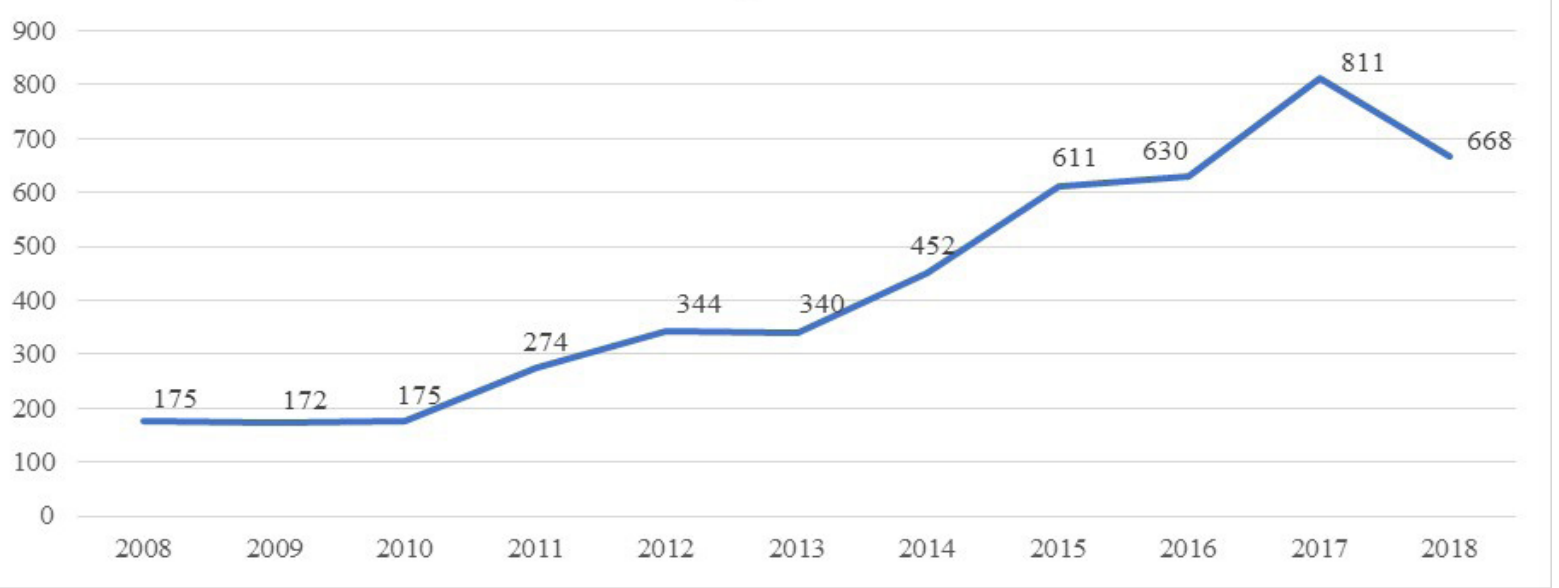

Fonte: Elaborado pelos autores (2018) 
A responsabilidade social associada à ecoinovação ainda é um tema recente na literatura científica. Geralmente a responsabilidade social está relacionada às ações sociais para com os colaboradores e a sociedade, além da filantropia, reputação e imagem organizacional (JAMALI; EL DIRANI; HARWOOD, 2015; VOEGTLIN; GREENWOOD, 2016).

Conforme Beretta (2018), é necessário saber se os projetos com objetivos ambientais também produzem resultados benéficos do ponto de vista social, com especial referência à questão da inclusão social. Ainda de acordo com a autora, A União Europeia está institucionalizando a ecoinovação e smart cities, visando a melhoria da qualidade de vida dos cidadãos.

A responsabilidade social com foco em inovação impulsiona a mudança organizacional, estimula uma gestão estratégica holística, abordagem para os desafios da sustentabilidade (ROOME, 2011). Neste cenário, Rake e Grayson (2009) destacam que a responsabilidade social pode atuar como um catalisador para lidar com problemas sociais urgentes que, se gerenciados adequadamente, podem ser transformados em oportunidades sociais de grande escala.

Recentes e drásticas mudanças socioeconômicas e políticas, ineficiências no setor público, recursos limitados devido à instabilidade macroeconômica (recessão econômica de 2008/2009) fazem com que as empresas se envolvam mais ativamente na mitigação de pressões ambientais e sociais, problemas além da mera gestão de externalidades, ocasionando uma criação de valor para o bem comum (HALKOS; SKOULOUDIS, 2018).

\section{PROPOSIÇÃO DE UM FRAMEWORK DE ANÁLISE}

Com o objetivo de propor um Framework de análise dos fatores das múltiplas hélices de inovação e da ecoinovação que contribua para o desenvolvimento sustentável, foi utilizada, como arcabouço teórico, a tríplice hélice, por entender que ela oferece respaldo para os principais conceitos da pesquisa (Figura 2). Entretanto, enquanto a maioria dos estudos da tríplice hélice aderem aos tradicionais atores institucionais (universidade, empresa e governo), é necessário expandir a perspectiva, e subdividir a indústria em grandes, médias e pequenas empresas, além de outra subdivisão em empresas de capital de risco, capital nacional ou internacional, pois as empresas têm diferentes características de rede de P\&D (LEE; KIM, 2016).

Contudo, muitos países, como parte de sua estratégia de inovação, experimentam uma mistura de funções e instituições, por meio de diversos acordos, como alianças estratégicas entre empresas, spin-off universitárias, parques tecnológicos e incubadoras tecnológicas para estimular inovações (ETZKOWITZ; DZISAH, 2007). 
De acordo com Severo (2013), para o desenvolvimento da inovação de produto e processo, ocorre a participação tanto de agentes internos como externos. Segundo a autora, no caso do Arranjo Produtivo Local Metalmecânico Automotivo (APLMMA) da Serra Gaúcha, a partir da necessidade de clientes ocorreu a parceria com fornecedores, a qual resultou em um novo material que gerou patente. Outro exemplo foi a participação do cliente e de consultoria externa para o desenvolvimento da inovação de produto e processo. Consoante isso, percebe-se uma coparticipação entre diferentes agentes para o desenvolvimento das inovações em organizações de diferentes portes e segmentos de atuação.

As hélices de inovação, indústria, universidade e governo, tradicionalmente reconhecidas na literatura (ETZKOWITZ; LEYDESDORFF, 1999; ETZKOWITZ; LEYDESDORFF, 2000), são fundamentais para o desenvolvimento das inovações. Entretanto, emergem outros agentes fundamentais associados às universidades, sendo os parques tecnológicos, spin-offs, incubadoras e startups. De acordo com Mengue, Schmidt e Bohnenberger (2019), as startups necessitam de aporte de diversos tipos de recursos para iniciarem suas atividades, principalmente financeiros. Conforme Nesello et al. (2019), embora as inovações estejam sendo amplamente discutidas e apoiadas, a sobrevivência das startups brasileiras depende da criação de mecanismos que fomentem a integração e geração de negócios entre seus múltiplos participantes.

No caso das empresas, os acionistas, equipes de consultorias e apoio, Organizações não Governamentais (ONGs), fornecedores e clientes podem também contribuir para o desenvolvimento das inovações. Pesquisas apontam para a necessidade não apenas de desenvolver os atuais sistemas de inovação, incluindo mais atores, bem como a preocupação com o meio ambiente, tratando-se de uma forma para alcance da sustentabilidade (GRUNDEL, 2016).

Neste cenário, a inovação com foco na sustentabilidade vem sendo amplamente discutida na literatura, com abordagem transdisciplinar, sendo reconhecida como inovações sustentáveis, inovações ecológicas, inovações verdes e ecoinovação (BOONS; LÜDEKE-FREUND, 2013; DÍAZ-GARCÍA; GONZÁLEZMORENO; SÁEZ-MARTÍNEZ, 2015; BOSSLE et al., 2016; CHEN et al., 2018).

Por conseguinte, a pressão competitiva é a principal força motriz na adoção da ecoinovação, seguida por instrumentos baseados no mercado, capacidades tecnológicas, demanda de clientes verdes e capacidade de organização ambiental (CAl; LI, 2018). Ecoinovação não é apenas desencadeada pela pressão institucional, mas também pelos recursos internos da empresa, e isso tem implicações para os governos e gestores de negócios. Coerentemente, caracteriza-se um modelo híbrido holístico de inovação e assim elenca-se a Proposição1:

Proposição 1: As hélices holísticas de inovação estão positivamente relacionadas à ecoinovação. 
Segundo Sato (2017), os riscos de mudança climática podem ser pelo menos parcialmente mitigados por uma abordagem transformacional, com novas ideias fomentadas e aprimoradas por universidades e empresas, em um ambiente de negócios apoiado por formuladores de políticas. Contudo, as práticas ambientais e ações sociais (DEY et al., 2018) podem estar associadas à ecoinovação, ocasionando resultados positivos para as organizações e sociedade, bem como uma melhor qualidade de vida para pessoas e gerações futuras (SEVERO; DE GUIMARÃES; DORION, 2018).

No que tange às práticas ambientais (MUÑOZ-VILLAMIZAR et al., 2018; PINTO et al., 2018), a segregação de resíduos, a coleta seletiva, a reciclagem, o sistema de gestão ambiental (SGA), a estação de tratamento de efluentes (ETE) (SEVERO; GUIMARÃES, 2015), a metodologia de P+L (YUSUP et al., 2015; SEVERO; DE GUIMARÃES; DORION, 2017; MATOS et al., 2018), as certificações ISO 14.001 e EMAS (Sistema Europeu de Ecogestão e Auditoria) (MURMURA et al., 2018) e as energias limpas (CAl; SAM; CHANG 2018) são primordiais para a sustentabilidade ambiental e estão se tornando práticas crescentes entre empresas nacionais e multinacionais.

Entretanto, conforme sugerem Pinto et al. (2018), no caso do BRICS (China, Índia, África do Sul e Brasil), vem-se estudando diversas práticas e soluções para as emissões de $\mathrm{CO}_{2}$. Além disso, nos estudos sobre a China, Índia e Brasil, as práticas de gestão ambiental abordaram diferentes tópicos, tais como o fornecimento e qualidade da água, os padrões regulatórios de gestão ambiental e as práticas do Green

\section{Supply Chain Management.}

No âmbito da responsabilidade social, a qual impacta a sociedade, pode-se considerar as ações governamentais por meio de políticas públicas, assim como as empresariais, visto que diversas ações sociais beneficiam pessoas que estão em vulnerabilidade social. Dentre as ações, elenca-se o auxílio financeiro para organizações sociais, doação de produtos e alimentos, campanhas de doação envolvendo os colaboradores, palestras e campanhas de conscientização sobre o meio ambiente (VOEGTLIN; GREENWOOD, 2016; SANTOS; SEVERO, 2018; HALKOS; SKOULOUDIS, 2018).

Neste cenário, organizações com práticas ambientais e ações sociais (DEY et al., 2018; LOOSEMORE et al. 2018) estão colhendo oportunidades de negócios, relacionadas às barreiras de crescimento sustentável (pobreza, mudança demográfica, mudança climática, escassez de recursos) (HALKOS; SKOULOUDIS, 2018). Consoante isso, incluir os pilares ambiental, econômico e social à ecoinovação pode ser considerada uma mudança de paradigma, uma nova filosofia relacionada à inovação (BOSSLE et al., 2016). Perante o exposto, elenca-se a Proposição 2:

Proposição 2: As práticas ambientais e ações sociais estão positivamente relacionadas à ecoinovação. 


\section{Gestãoe \\ Desenvolvimento}

e-ISSN: 2446-6875

p-ISSN: $1807-5436$

Contudo, após a formulação das duas proposições (P1 e P2), emerge a contribuiç̧ão da ecoinovação para o desenvolvimento regional, assim como para as smart cities, uma vez que as cidades inteligentes são projetos nos quais um determinado espaço urbano utiliza tecnologias de comunicação e informação sensíveis ao contexto, de gestão urbana e ação social dirigidos por dados (CARAGLIU; DEL BO; NIJKAMP, 2011; FEl et al., 2016), com referência à questão da inclusão social (BERETTA, 2018).

De acordo com Beretta (2018), as populações urbanas estão aumentando, assim como a carga sobre a energia, transporte, água, edifícios e espaços públicos. Neste cenário, as smart cities representam o contexto em que ecoinovação é altamente necessária. Para tanto, é necessário encontrar soluções inteligentes, ou seja, altamente eficientes e sustentáveis, gerando prosperidade econômica e bemestar social. Ressalta-se que o compartilhamento de recursos comuns com o objetivo de estabelecer ecossistemas de inovação urbanos e regionais requer parcerias sustentáveis e estratégias de cooperação entre os diferentes stakeholders, os quais visam ao desenvolvimento sustentável. Tal desenvolvimento é capaz de atender às necessidades das gerações atuais sem comprometer a capacidade de suprimentos das gerações futuras.

Figura 2 - Framework de análise dos fatores das múltiplas hélices de inovação e da ecoinovação que contribuem para o desenvolvimento sustentável

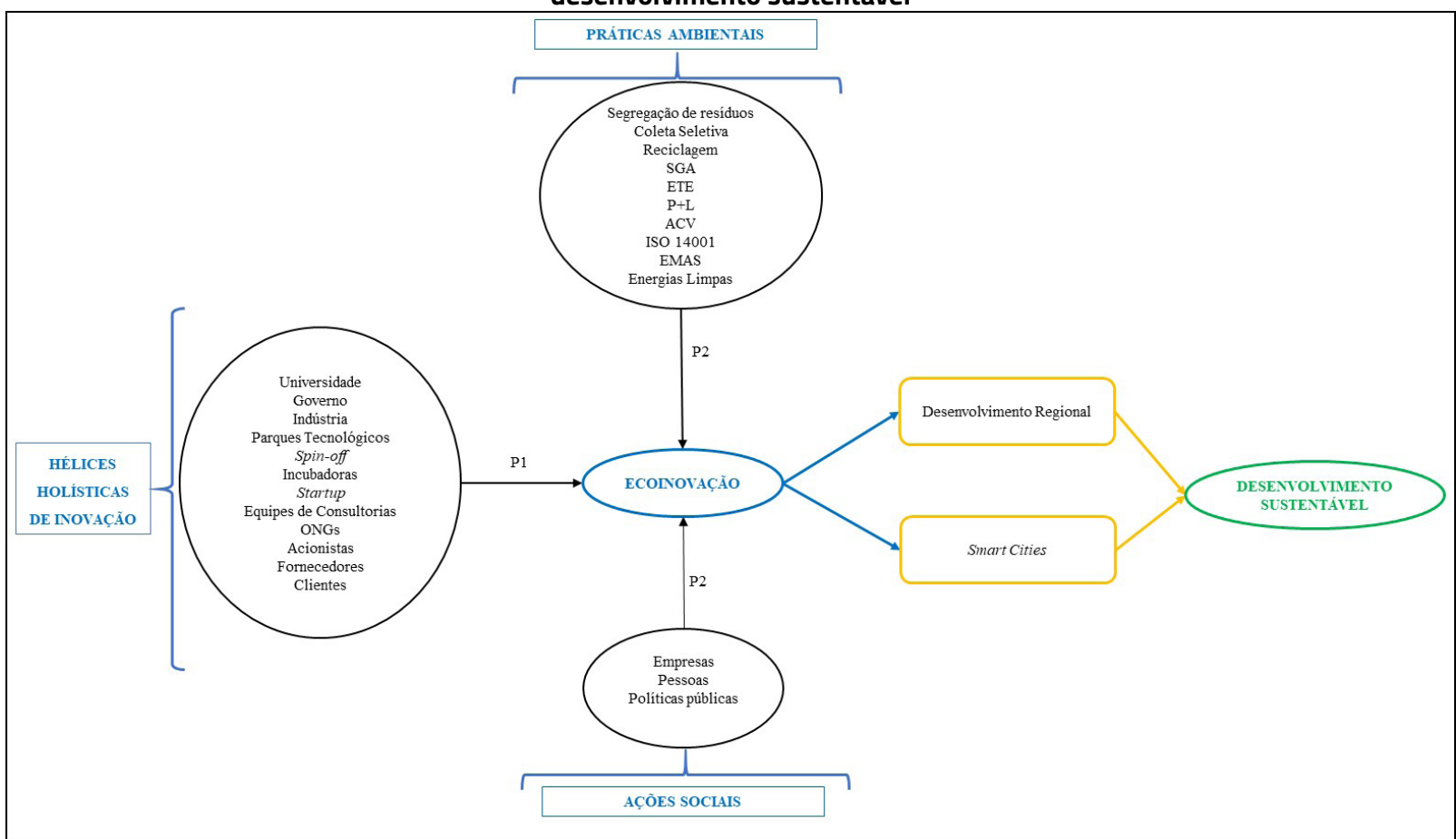

Fonte: Elaborado pelos autores (2018) 


\section{CONSIDERAÇÕES FINAIS}

Considerando a perspectiva de avançar a compreensão sobre a relação entre as múltiplas hélices de inovação e a ecoinovação, apresentou-se um Framework na perspectiva do desenvolvimento sustentável. No âmbito das múltiplas hélices, compõe-se um modelo híbrido holístico de inovação, onde destacam-se: indústria, universidade, governo, parques tecnológicos, spin-offs, incubadoras de empresas, startups, fornecedores, clientes, ONGs e acionistas, os quais contribuem para a ecoinovação. Perante o exposto, elegeu-se a Proposição 1: As Hélices holísticas de inovação estão positivamente relacionadas à ecoinovação.

No que tange a práticas ambientais que contribuem para a ecoinovação elencam-se: segregação de resíduos, coleta seletiva, reciclagem, SGA, ETE, P+L, ACV, ISO 14001, EMAS e energias limpas. Já as ações sociais estão ligadas às políticas públicas, pessoas e empresas. Coerentemente, a partir destes achados, apresenta-se a Proposição 2: As práticas ambientais e ações sociais estão positivamente relacionadas à ecoinovação.

O ensaio teórico se justifica na medida em que os temas representam uma lacuna na literatura, com escassos trabalhos que versam especificamente sobre as relações das múltiplas hélices de inovação sobre a ecoinovação, assim como das práticas ambientais e ações sociais sobre a ecoinovação, visto que, em muitos artigos, as temáticas são pesquisadas separadamente. Nesse sentido, as contribuições gerenciais estão atreladas às informações das diferentes hélices de inovação que podem ser utilizadas pelos gestores, para fomentar uma inovação que contribua para o desenvolvimento sustentável, assim como as práticas ambientais e ações socias que visam à preservação do meio ambiente e uma melhor qualidade de vida das pessoas e do planeta.

Como contribuições acadêmicas, a pesquisa desenvolveu um Framework teórico que poderá ser utilizado em outras pesquisas, bem como aplicado no contexto organizacional e testado por meio de estudos empíricos. Com relação às contribuições para o avanço do conhecimento, destacam-se as diversas múltiplas hélices de inovação que contribuem para a ecoinovação. Entretanto, é necessário que estas hélices estejam articuladas entre si, pois separadamente não contribuirão para o desenvolvimento regional, a geração de renda e empregos, os quais são fundamentais para impactar a melhoria da qualidade de vida das pessoas, a utilização racional dos recursos naturais e a diminuição do impacto ambiental no meio ambiente.

Quanto às limitações, estas estão atreladas à Base de dados utilizada(Science Direct), assim, sugeremse novas pesquisas, com outras bases de dados (SCOPUS, EBSCO), bem como estudos qualitativos para a obtenção de informações in loco sobre os benefícios das múltiplas hélices de inovação, das práticas ambientais e ações sociais. 


\section{Gestãoe \\ Desenvolvimento}

e-ISSN: 2446-6875

p-ISSN: $1807-5436$

\section{REFERÊNCIAS}

BARDIN, L. Análise de conteúdo. Lisboa: Edições 70, 2011.

BERETTA, I. The social effects of eco-innovations in Italian smart cities. Cities, v. 72, p. 115-121, 2018.

BOSCH-SIJTSEMA, P. M.; BOSCH, J. Plays nice with others? Multiple ecosystems, various roles and divergent engagement models. Technology Analysis \& Strategic Management, v. 27, n. 8, p. 960-974, 2015.

BOSSLE, M. B.; de BARCELLOS, M. D.; VIEIRA, L. M.; SAUVÉE, L. The drivers for adoption of eco-innovation. Journal of Cleaner Production, 113, 861-872, 2016.

BOONS, F.; LÜDEKE-FREUND, F. Business models for sustainable innovation: state-of-the-art and steps towards a research agenda. Journal of Cleaner Production, v. 45, p. 9-19, 2013.

BREM, A.; RADZIWON, A. Efficient Triple Helix collaboration fostering local niche innovation projects - A case from Denmark. Technological Forecasting and Social Change, v. 123, p. 130-141, 2017.

CAI, W.; LI, Gi. The drivers of eco-innovation and its impact on performance: Evidence from China. Journal of Cleaner Production, v. 176, p. 110-118, 2018.

CAI, Y.; SAM, C. Y.; CHANG, T. Nexus between clean energy consumption, economic growth and CO2 emissions. Journal of Cleaner Production, v. 182, p. 1001-1011, 2018.

CANCINO, C. A.; LA PAZ, A. I.; RAMAPRASAD, A.; SYN, T. Technological innovation for sustainable growth: An ontological perspective. Journal of Cleaner Production, 179, 31-41, 2018.

CARAGLIU, A.; DEL BO, C.; NIJKAMP, P. Smart cities in Europe. Journal of Urban Technology, v. 18, n. 2, p. 65-82, 2011

CARAYANNIS, E. G.; CAMPBELL, D. F J. 'Mode 3'and'Quadruple Helix': toward a 21st century fractal innovation ecosystem. International Journal ff Technology Management, v. 46, n. 3-4, p. 201-234, 2009.

CARAYANNIS, E. G.; CAMPBELL, D. FJ. Triple helix, quadruple helix and quintuple helix and how do knowledge, innovation and the environment relate to each other?: a proposed framework for a trans-disciplinary analysis of sustainable development and social ecology. International Journal of Social Ecology and Sustainable Development, v. 1, n. 1, p. 41-69, 2010.

CARAYANNIS, E. G.; CHEREPOVITSYN, A. E.; ILINOVA, A. A. Sustainable development of the Russian arctic zone energy shelf: the role of the quintuple innovation helix model. Journal of the Knowledge Economy, v. 8, n. 2, p. 456-470, 2017. 


\section{Gestãoe \\ Desenvolvimento}

e-ISSN: 2446-6875

p-ISSN: $1807-5436$

CHEN, J.; CHENG, J.; DAl, S. Regional eco-innovation in China: An analysis of eco-innovation levels and influencing factors. Journal of Cleaner Production, v. 153, p. 1-14, 2017.

CHEN, X.; YI, N.; ZHANG, L.; LI, D. Does institutional pressure foster corporate green innovation? Evidence from China's top 100 companies. Journal of Cleaner Production, v. 188, p. 304-311, 2018.

CHENG, C. C.; SHIU, E. C. Validation of a proposed instrument for measuring eco-innovation: An implementation perspective. Technovation, v. 32, n. 6, p. 329-344, 2012.

CHENG, C. C.; YANG, C-L.; SHEU, C. The link between eco-innovation and business performance: a Taiwanese industry context. Journal of Cleaner Production, v. 64, p. 81-90, 2014.

CORREIA, S. E. N.; GOMÉZ, C. R. P.; OLIVEIRA, V. M. Inovações sociais e seus níveis de análise: o caso PATAC-PB. Revista Gestão e Desenvolvimento, v. 14, n. 2, p. 157-180, 2018.

CRESWELL, J. W. Projeto de pesquisa métodos qualitativo, quantitativo e misto. 3. ed. Porto Alegre: Artmed, 2010.

DA SILVA, O. T.; CHAIS, C.; RADAELLI, A. A. P.; GANZER, P. P.; D'AVILA, A. A. F.; OLEA, P. M.; DORION, E. C. H.; PRODANOV, C. C.; DA CRUZ, M. R. Inovação em pequena propriedade rural: uma perspectiva teórica. Revista Gestão e Desenvolvimento, v. 14, n. 1, p. 34-44, 2017.

DEY, P. K.; PETRIDIS, N. E.; PETRIDIS, K.; MALESIOS, C.; NIXON, J. D.; GHOSH, S. K. Environmental management and corporate social responsibility practices of small and medium-sized enterprises. Journal of Cleaner Production, v. 195, p. 687-702, 2018.

DÍAZ-GARCÍA, C.; GONZÁLEZ-MORENO, Á.; SÁEZ-MARTÍNEZ, F. J. Eco-innovation: insights from a literature review. Innovation, v. 17, n. 1, p. 6-23, 2015.

DUDIN, M. N.; FROLOVA, E. E.; GRYZUNOVA, N. V.; SHUVALOVA, E. B. The Triple helix model as a mechanism for partnership between the state, business, and the scientific-educational community in the area of organizing national innovation development. Asian Social Science, v. 11, n. 1, p. 230-238, 2015.

ETZKOWITZ, H. Innovation in innovation: the Triple Helix of university-industry government relations. Social Science Information, v. 42, p. 293-337, 2003a.

ETZKOWITZ, H. Research groups as "quasi-firms": the invention of the entrepreneurial university. Research Policy, v. 32, p. 109-121, 2003 b. 


\section{Gestãoe \\ Desenvolvimento}

e-ISSN: 2446-6875

p-ISSN: 1807-5436

ETZKOWITZ, H.; DZISAH, J. The triple helix of innovation: towards a university-led development strategy for Africa. ATDF Journal, v. 4, n. 2, p. 3-10, 2007.

ETZKOWITZ, H.; LEYDESDORFF, L. The triple helix-university-industry-government relations: a laboratory for knowledge-based economic development. European Association Study Science and Technology Review, v. 14, n. 1, p. 14-19, 1995.

ETZKOWITZ, H.; LEYDESDORFF, L. The dynamics of innovation: from National Systems and "Mode 2" to a Triple Helix of university-industry-government relations. Research Policy, v. 29, n. 2, p. 109-123, 2000.

ETZKOWITZ, H.; LEYDESDORFF, L. Whose triple helix? Science and Public Policy, v. 26, n. 2, p. 138-139, 1999.

ETZKOWITZ, H.; ZHOU, C. Hélice Tríplice: inovação e empreendedorismo universidade-indústria-governo. Estudos Avançados, v. 31, n. 90, p. 23-48, 2017.

FEl, J.; WANG, Y.; YANG, Y.; CHEN, S.; ZHI, Q. Towards eco-city: The role of green innovation. Energy Procedia, v. 104, p. 165-170, 2016.

FLICK, U. Introdução à pesquisa qualitativa. 3. ed. Porto Alegre: Artmed, 2009.

GARCÍA-GRANERO, E. M.; PIEDRA-MUÑOZ, L.; GALDEANO-GÓMEZ, E. Eco-innovation measurement: A review of firm performance indicators. Journal of Cleaner Production, v. 191, p. 304-317, 2018.

GIBBS, G. Análise de dados qualitativos: coleção pesquisa qualitativa. Porto Alegre: Bookman Editora, 2009.

GIL, A. C. Como elaborar projetos de pesquisa. 5. ed. São Paulo: Atlas, 2010.

GOUVEA, R.; KASSICIEH, S.; MONTOYA, M. J. R. Using the quadruple helix to design strategies for the green economy. Technological Forecasting and Social Change, v. 80, n. 2, p. 221-230, 2013.

GRUNDEL, I.; DAHLSTRÖM, M. A Quadruple and Quintuple Helix approach to regional innovation systems in the transformation to a forestry-based bioeconomy. Journal of the Knowledge Economy, v. 7, n. 4, p. 963-983, 2016.

GUERRERO, M.; URBANO, D. The impact of Triple Helix agents on entrepreneurial innovations' performance: An inside look at enterprises located in an emerging economy. Technological Forecasting and Social Change, v. 119, p. 294-309, 2017. 


\section{Gestãoe \\ Desenvolvimento}

e-ISSN: 2446-6875

p-ISSN: 1807-5436

HALILA, F.; RUNDQUIST, J. The development and market success of eco-innovations: A comparative study of eco-innovations and "other" innovations in Sweden. European Journal of Innovation Management, v. 14, n. 3, p. 278-302, 2011.

HALKOS, G.; SKOULOUDIS, A. Corporate social responsibility and innovative capacity: Intersection in a macro-level perspective. Journal of Cleaner Production, v. 182, p. 291-300, 2018.

HOJNIK, J.; RUZZIER, M. What drives eco-innovation? A review of an emerging literature. Environmental Innovation and Societal Transitions, v. 19, p. 31-41, 2016.

HOJNIK, J.; RUZZIER, M.; MANOLOVA, T. S. Internationalization and economic performance: The mediating role of eco-innovation. Journal of Cleaner Production, v. 171, p. 1312-1323, 2018.

JAMALI, D. R.; EL DIRANI, A. M.; HARWOOD, I. A. Exploring human resource management roles in corporate social responsibility: the CSR HRM co creation model. Business Ethics: A European Review, v. 24, n. 2, p. 125-143, 2015.

KUO, T-C.; SMITH, S. A systematic review of technologies involving eco-innovation for enterprises moving towards sustainability. Journal of Cleaner Production, 192, p. 207-220, 2018.

LEE, Y. H.; KIM, Y. J. Analyzing interaction in R\&D networks using the Triple Helix method: Evidence from industrial R\&D programs in Korean government. Technological Forecasting and Social Change, v. 110, p. 93-105, 2016.

LI, D.; ZHAO, Y.; ZHANG, L.; CHEN, X.; CAO, C. Impact of quality management on green innovation. Journal of Cleaner Production, v. 170, p. 462-470, 2018.

LI, Y.; ARORA, S.; YOUTIE, J.; SHAPIRA, P. Using web mining to explore Triple Helix influences on growth in small and mid-size firms. Technovatin, In press, 2016.

LIU, Y.; HUANG, Q. University capability as a micro-foundation for the Triple Helix model: The case of China. Technovation, In press, 2018.

LOOSEMORE, M.; LIM, B. T. H.; LING, F. Y. Y.; ZENG, H. Y. A comparison of corporate social responsibility practices in the Singapore, Australia and New Zealand construction industries. Journal of Cleaner Production, v. 190, p. 149-159, 2018.

LÓPEZ, F. J. D.; MONTALVO, C. A comprehensive review of the evolving and cumulative nature of eco-innovation in the chemical industry. Journal of Cleaner Production, v. 102, p. 30-43, 2015. 


\section{Gestãoe \\ Desenvolvimento}

e-ISSN: 2446-6875

p-ISSN: 1807-5436

MATOS, L. M.; ANHOLON, R.; DA SILVA, D.; ORDOÑEZ, R. E. C.; QUELHAS, O. L. G.; LEAL FILHO, W.; DE SANTA-EULALIA, L. A. Implementation of cleaner production: A ten-year retrospective on benefits and difficulties found. Journal of Cleaner Production, 187, 409-420, 2018.

MENGUE, T. H. S.; SCHMIDT, S.; BOHNENBERGER, M. C. Contribuições do investimento anjo para o desenvolvimento de startups na região metropolitana de Porto Alegre. Revista Gestão e Desenvolvimento, v. 16, n. 1, p. 71-98, 2019.

MUÑOZ-VILLAMIZAR, A.; SANTOS, J.; VILES, E.; ORMAZÁBAL, M. Manufacturing and environmental practices in the Spanish context. Journal of Cleaner Production, v. 178, p. 268-275, 2018.

MURMURA, F.; LIBERATORE, L.; BRAVI, L.; CASOLANI, N. Evaluation of Italian companies' perception about ISO 14001 and eco management and audit scheme III: motivations, benefits and barriers. Journal of Cleaner Production, v. 174, p. 691-700, 2018.

NESELLO, P.; GUERRA, R. M. A.; MACEDO, T. M.; GANZER, P. P.; CAMARGO, M. E.; OLEA, P. M. Criação de spin-offs acadêmicos: uma análise dos critérios determinantes de desempenho. Revista Gestão e Desenvolvimento, v. 16, n. 3, p. 33-57, 2019.

NILSSEN, M. To the smart city and beyond? Developing a typology of smart urban innovation. Technological Forecasting and Social Change, In Press. 2018.

PIALOT, O.; MILLET, D. Towards operable criteria of eco-innovation and eco-ideation tools for the early design phases. Procedia CIRP, v. 69, p. 692-697, 2018.

PINTO, G. M. C.; PEDROSO, B.; MORAES, J.; PILATTI, L. A.; PICININ, C. T. Environmental management practices in industries of Brazil, Russia, India, China and South Africa (BRICS) from 2011 to 2015. Journal of Cleaner Production, v. 198, p. 1251-1261, 2018.

RAKE, M.; GRAYSON, D. Embedding corporate responsibility and sustainability-everybody's business. Corporate Governance: The International Journal of Business in Society, v. 9, n. 4, p. 395-399, 2009.

RAZAK, A. A.; WHITE, G. The triple helix model for innovation: a holistic exploration of barriers and enablers. International Journal of Business Performance and Supply Chain Modelling, v. 7, n. 3, p. 278291, 2015.

ROHRBECK, R.; HÖLZLE, K.; GEMÜNDEN, H. G. Opening up for competitive advantage-How Deutsche Telekom creates an open innovation ecosystem. R\&D Management, v. 39, n. 4, p. 420-430, 2009. 


\section{Gestãoe \\ Desenvolvimento}

e-ISSN: 2446-6875

p-ISSN: 1807-5436

ROOME, N. A retrospective on globalization and sustainable development: the business challenge of systems organization and systems integration. Business \& Professional Ethics Journal, v. 30, n. 3-4, p. 195-230, 2011.

RYAN, P.; GEOGHEGAN, W.; HILLIARD, R. The microfoundations of firms' explorative innovation capabilities within the triple helix framework. Technovation, In Press, 2018.

SANTOLARIA, M.; OLIVER-SOLÀ, J.; GASOL, C. M.; MORALES-PINZÓN, T.; RIERADEVALL, J. Eco-design in innovation driven companies: perception, predictions and the main drivers of integration. The Spanish example. Journal of Cleaner Production, v. 19, n. 12, p. 1315-1323, 2011.

SANTOS, J.; SEVERO, E. A. Responsabilidade social das empresas para as práticas das organizações da sociedade civil em cidades do Norte do Rio Grande do Sul. Revista Ciências Administrativas, v. 24, p. $1-16,2018$

SATO, S. Climate change, the built environment and triple-helix innovation. Energy Procedia, v. 143, p. 843-850, 2017.

SEVERO, E. A.; GUIMARAES, J. C. F. Corporate environmentalism: an empirical study in Brazil. International Journal of Business and Globalisation, v. 15, p. 81-95, 2015.

SEVERO, E. A.; DE GUIMARÃES, J. C. F.; DORION, E. C. H. Cleaner production, social responsibility and eco-innovation: Generations' perception for a sustainable future. Journal of Cleaner Production, v. 186, p. 91-103, 2018.

SEVERO, E. A.; DE GUIMARÃES, J. C. F.; DORION, E. C. H. Cleaner production and environmental management as sustainable product innovation antecedents: A survey in Brazilian industries. Journal of Cleaner Production, v. 142, p. 87-97, 2017.

TAMAYO-ORBEGOZO, U.; VICENTE-MOLINA, M-A.; VILLARREAL-LARRINAGA, O. Eco-innovation strategic model. A multiple-case study from a highly eco-innovative European region. Journal of Cleaner Production, v. 142, p. 1347-1367, 2017.

TRENCHER, G. P.; YARIME, M.; KHARRAZI, A. Co-creating sustainability: cross-sector university collaborations for driving sustainable urban transformations. Journal of Cleaner Production, v. 50, p. 40-55, 2013

VAIVODE, I. Triple Helix model of university-industry-government cooperation in the context of uncertainties. Procedia-Social and Behavioral Sciences, v. 213, p. 1063-1067, 2015. 


\section{Gestãoe \\ Desenvolvimento}

e-ISSN: 2446-6875

p-ISSN: 1807-5436

VOEGTLIN, C.; GREENWOOD, M. Corporate social responsibility and human resource management: A systematic review and conceptual analysis. Human Resource Management Review, v. 26, n. 3, p. 181197, 2016.

XAVIER, A. F.; NAVEIRO, R. M.; AOUSSAT, A.; REYES, T. Systematic literature review of eco-innovation models: Opportunities and recommendations for future research. Journal of Cleaner Production, v. 149, p. 1278-1302, 2017.

YANG, Y.; HOLGAARD, J. E.; REMMEN, A. What can triple helix frameworks offer to the analysis of eco-innovation dynamics? Theoretical and methodological considerations. Science and Public Policy, v. 39, n. 3, p. 373-385, 2012.

YUSUP, M. Z.; MAHMOOD, W. H. W.; SALLEH, M. R.; AB RAHMAN, M. N. The implementation of cleaner production practices from Malaysian manufacturers' perspectives. Journal of Cleaner Production, $\mathrm{V}$. 108, p. 659-672, 2015. 